\title{
Féeries
}

Études sur le conte merveilleux, XVII ${ }^{\mathrm{e}} \mathrm{XIX} \mathrm{X}^{\mathrm{e}}$ siècle

\section{Robert Irwin, The Arabian Nights. A Companion}

London, Allen Lane, The Penguin Press, 1994, 344 p.

Jean-Paul Sermain

\section{OpenEdition}

Journals

Édition électronique

URL : http://journals.openedition.org/feeries/89

DOI : 10.4000/feeries.89

ISSN : 1957-7753

Éditeur

UGA Éditions/Université Grenoble Alpes

Édition imprimée

Date de publication : 1 février 2004

Pagination : 202-205

ISSN : 1766-2842

Référence électronique

Jean-Paul Sermain, «Robert Irwin, The Arabian Nights. A Companion », Féeries [En ligne], 1 | 2004, mis en ligne le 29 janvier 2007, consulté le 24 septembre 2020. URL : http://journals.openedition.org/ feeries/89; DOI : https://doi.org/10.4000/feeries.89

Ce document a été généré automatiquement le 24 septembre 2020

(c) Féeries 


\section{Robert Irwin, The Arabian Nights. A Companion}

London, Allen Lane, The Penguin Press, 1994, 344 p.

Jean-Paul Sermain

1 En attendant la publication de la nouvelle traduction des Mille et Une Nuits par A. Miquel et J.E. Bencheikh dans la collection de la Pléiade (dont la moitié a été publiée en Folio mais sans apparat critique), en attendant l'ouvrage de A. Chraïbi et la réédition de l'édition Galland en GF, ce volume constitue la meilleure introduction aux Mille et Une Nuits, conformément à ce que les Anglais appellent un " companion» (on notera que le meilleur ouvrage sur Don Quichotte, celui de A. Riley, vient d'Angleterre, les Espagnols l'ont traduit: il n'en est pas trace en France...). Le public visé explique que l'auteur donne une part importante aux traductions en anglais et aux travaux érudits dans cette langue, mais il fait part égale avec les traductions et travaux français. La date d'achèvement du manuscrit (vers 1990) l'empêche de tenir compte des travaux plus récents de l'équipe française autour d'A. Miquel, C. Brémond et J.E. Bencheikh. Le livre ne comporte pas de bibliographie, mais les notes donnent pour les différents chapitres des indications très abondantes: le principe $\mathrm{du}$ " companion » est que l'auteur évalue l'utilité ou la justesse des travaux et indique les profits que le lecteur peut tirer des différents ouvrages qu'il mentionne. Le livre n'est pas une étude des Mille et Une Nuits mais recense les perspectives successives dans lesquelles ont peut les envisager dans la mesure où il ne s'agit pas d'un texte fixe mais d'une compilation aux états multiples, étalés sur plus de dix siècles, dont les contours sont donc variables et dont l'ensemble n'est pas encore connu précisément. L'auteur présente successivement neuf états de la question qu'on peut regrouper en trois groupes. Il dessine à l'horizon un paradoxe inévitable : l'idéal d'une édition critique qui, pour chaque conte, s'appuierait sur les manuscrits existants, tenterait d'établir la meilleure édition puis examinerait les liens de chaque conte avec les diverses traditions dans lesquelles il figure, détruit à terme les Mille et une nuits : celles-ci ne peuvent exister que par des choix limitatifs, par certains côtés arbitraires. La connaissance précise des contes se fera au détriment des Mille et 
une nuits, et celle ci n'existent qu'en sacrifiant cet accès le plus informé et le plus juste aux contes.

Trois types d'approches donc. Le premier, peut-être le plus important pour la connaissance du texte, occupe les trois premiers chapitres et concerne l'état des connaissances sur le texte où les problèmes d'édition et de traductions sont indissociables. En effet, la première édition des nuits est la traduction de Galland, basée pour une part sur un manuscrit aujourd'hui à la Bibliothèque nationale et qui se révélera le plus ancien existant ( $\mathrm{XIV}^{\mathrm{e}}-\mathrm{XV}^{\mathrm{e}}$ siècle), et sur des variées écrites et orales... Ce début (qui va connaître son tricentenaire) ouvre une période de chasse aux manuscrits, d'enquêtes archéologiques, d'éditions et de traductions. Le texte des Mille et Une Nuits ne peut être séparé de son histoire : les éditions en arabe ont dû attendre l'introduction de l'imprimerie au XIX ${ }^{\mathrm{e}}$ siècle, elles viennent du Caire et de Calcutta. La seconde faite en ce lieu (due à MacNaghten) est la plus complète, mais aucune n'établit ses sources et n'explicite ses choix. La première édition scientifique en arabe est tardive : elle a été faite aux États-Unis par un chercheur, Mahdi, qui a réalisé le projet du grand Mac Donald: c'est une édition du manuscrit Galland qui a collationné l'ensemble des manuscrits syriens voisins de façon à établir leur généalogie et à remonter à un texte "mère " qui serait syrien du xiIIe siècle. Une seconde enquête consiste à essayer d'établir les éléments de la genèse du texte en rapportant en même temps l'histoire de cette progressive découverte : des recueils intitulés « Mille contes ", puis « Mille nuits » et enfin "Mille et Une Nuits " sont évoqués à partir du IX siècle, définis par le récit cadre. On peut considérer la famille des manuscrits syriens comme le point d'aboutissement de cette première histoire. Une seconde famille qui complète ce manuscrit en faisant feu de tout bois se développe en Égypte à la fin $d u$ XVIII $^{\mathrm{e}}$ siècle jusqu'au début du XIX ${ }^{\mathrm{e}}$ siècle : ce sont ces textes tardifs et copieux (arrivant enfin à 1001 Nuits) qui sont édités en arabe puis traduits en Europe. Cette troisième étape de la diffusion en prépare une quatrième en cours de réalisation: l'établissement pour chaque conte d'une édition critique. Un troisième chapitre replace les Mille et Une Nuits dans la millénaire et mobile culture du conte: il relève des versions parallèles et anciennes de plusieurs des contes, il étudie les transferts possibles, les migrations, les influences. Tout conte apparaît ainsi comme une version dans un ensemble polymorphe, toujours en voie de transformation par coupures, ajouts, condensations, expansion de contes antérieurs, voisins, préparant d'autres contes dans une prolifération qui s'achève au moment où un manuscrit sert de canon et passe à l'imprimé: le livre marque progressivement la fin de cette littérature narrative de sagesse (achevée chez nous avec La Fontaine). Quand Galland traduit les Mille et Une Nuits, le lecteur est en train de s'en détacher : leur donner une forme définitive (comme pour les contes de fées contemporains), c'est assurer leur survie et déclarer leur mort.

3 Le deuxième tiers du livre, qui correspond aux cinq chapitres suivants, envisage divers «thèmes » des Mille et Une Nuits et apprécie leur traitement en fonction de leur degré de pertinence et de leur qualité informative sur les mœurs ou les conceptions du monde arabe (dans une longue durée...). Pour cela, il opère une confrontation avec les témoignages contemporains ou les enquêtes des historiens. Ainsi pour les questions de l'art du conteur, des divertissements des rues, de la vie des bas-fonds, des intrigues sexuelles, ou des merveilles, l'auteur recense les textes les plus anciens, par exemple poésie érotique, livres de gueux, livres de magie, livres de curiosités. Mais il recense aussi les spectacles qui pouvaient être proposés au public des grandes villes, ou aux 
conditions d'action de la police et à son intégration éventuelle de bandes de malfrats. Il s'agit à la fois de retrouver derrière la fiction les realia alors évidentes, et de restituer les cadres mentaux et les normes par rapport auxquels on peut évaluer les comportements des personnages ou la conduite des contes. R. Irwin donne les éléments d'une littérature et d'une civilisation (ou les moyens de les retrouver), qui permettent de comprendre en partie le propos des auteurs des Mille et Une Nuits, la manière dont ils ont pu être appréciés (entendus sans doute et lus).

La troisième partie du livre rend compte des approches littéraires des Mille et Une Nuits. Elle se déroule en deux chapitres inégaux. Le premier, consacré aux «lectures formelles ", dresse un bilan plutôt maigre : en effet d'une part, la qualité littéraire n'est pas reconnue aux Mille et Une Nuits dans les pays de langue arabe, et les Européens se sentent contraints à n'aborder que la substance du conte, son matériau narratif, ce qu'il raconte. Aussi R. Irwin discute l'intérêt du modèle de Propp pour l'analyse du récit, mentionne à plusieurs reprises l'article de Todorov sur les hommes-récit (qui ne s'applique qu'à une partie du texte). Les travaux français s'orientent eux aussi pour une grande part (l'autre étant la recherche des realia telle que R. Irwin l'a présentée précédemment) vers l'analyse de la construction du conte, soit pour en affirmer la logique transcendante, comme J.-E. Bencheikh, soit en adaptant la logique des possibles narratifs sur le plan des transformations historiques comme le fait C. Brémond: en reconstituant une mémoire encyclopédique des contes, il peut interpréter chaque moment du conte comme un choix parmi ceux avérés par ailleurs. Le dernier chapitre du livre envisage ces lectures des Mille et Une Nuits que sont les diverses réécritures de son texte. Ce chapitre fait une part assez belle à la littérature de langue française du XVIII ${ }^{e}$ siècle : s'il écarte les Gueullette et les Hamilton comme les Voltaire et les Caylus (n'est pas posée ainsi la question d'une reprise ironique des contes arabes : contresens majeur mais rebondissant sur une autre littérature), il accorde une grande place à Cazotte, Beckford, ainsi qu'à Potocki et au roman gothique : lointains héritiers peutêtre. Le lecteur français découvrira les œuvres anglaises du XVIII et du $\mathrm{XIX}^{\mathrm{e}}$ assez étroitement inspirées par les Nuits, les analyses des textes de Borgès et de Barth, la France ne faisant une brève apparition au $\mathrm{xx}^{\mathrm{e}}$ siècle qu'avec Proust (on pourra consulter à ce sujet le livre postérieur de Dominique Jullien). 